\title{
MATHEMATICAL ANALYSIS OF FINGER POSITIONS OF A SPHERICAL LINKAGE METAMORPHIC ROBOT PALM
}

\author{
Mujibur Rahman Khan \\ Department of Mechanical Engineering, BUET, Bangladesh \\ and \\ Jian S. Dai \\ Kings College, University of London, UK
}

\begin{abstract}
Grasping is the main function performed by the mechanical hand. Mechanisms with more functionality are needed for secure grasping of different and complex objects. With the advancement of the field of robotics it is important to think of mechanisms of multifingered robot hand with added functionality. The metamorphic mechanism with spherical linkage opens a wide opportunity to design a multi fingered robot hand with spherical palm, which can achieve human like dexterity. On the basis of an intensive study of literature, this paper focuses on the development of a geometrical model and mathematical analysis of spherical robot palm with fingers attached to it. To develop the generalised mathematical model, the co-ordinate transformation matrices of the joint axis position and the finger position have been derived. The transformation matrices have been derived with the position and orientation of the links and finger positions. So the coordinate points of a point of interest in the three dimensional space can be described at different configuration state of the mechanism. The detail analysis has been done through this mathematical and geometrical modelling of five-bar spherical metamorphic linkage robotic palm. This mathematical and geometrical model can be used to inspect the feasibility of the mechanism design for a multi fingered robot palm.
\end{abstract}

\section{INTRODUCTION}

With added functionality like ability to grasp as well as manipulation, mulitifingered hand with human like metamorphic palm has the potential to increase the number of contact points with an objects for achieving more stable grasp. In addition, the ability to reach a wide range of grasp configurations will allow many more types of objects to be grasped properly.

There are other important features of multifingered robotic palms. If the object can be manipulated with in the hand, however, the palm, fingers, and the object it self must move. This approach of executing fine motion fine motion leads to better control. This kind of robotic hand will also be able to change the orientation of the grasped objects. Fingers can reach into restricted environments where arms cannot reach.

All these unique features and capabilities of multifingered robotic palm motivated the author to study, analysis and develop the mathematical model and geometrical formation of this kind of robot hand. This present analysis will be helpful for determining the amount of flexibility resulting form the simple mechanism and extending the classes of tasks possible with the mechanism.

\section{LIETRATURE REVIEW}

Study of multifingered robot hands centred on the development of the mechanism with mathematical and geometrical model, unified control system architecture for manipulation and sensor development. Romdhane and Duffy $^{1}$ presents a kinetic analysis of multifingered hands with secure grasp of general object plus the manipulation of this object by the fingers without motion of the arm on which it is mounted. A pair of matrices is introduced that relate the small motion of the joints to the relative displacement of the objects and the fingertips. In the work done by Salisbury ${ }^{2}$, which was subsequently extended by Kobayashi ${ }^{3}$, a pair of matrices describing the kinestatics (instantaneous motions and static) of hand was presented. Kerr and Sanger ${ }^{4}$ introduce a stiffness matrix for a grasp with elastic contacts. They deduced the internal force on the grasped object and determine weather it would slip.

In the Ph.D. thesis of Kerr ${ }^{5}$, it has been presented that multifingered hands will increase the abilities and overall flexibility of robot systems. This thesis explores some of the issues in the kinematics of manipulating objects with in a hand. The analysis presented is applicable to a wide variety of hands. The kinematic issues examined are the determination of the finger tip and finger joint motions required to create a desire motion of the object, identifying situations where objects cannot be manipulated with complete generality and determining the workspace of the hand.

Kerr and Roth $^{6}$ discuss three of the fundamental problems encountered in grasping and manipulation of objects with in a multifingered hand. The first of these problems is that of determining how hard to squeeze an object with the fingers in order to ensure that the object is grasped properly. This part of work follows closely on Salisbury and Craig ${ }^{7}$. The second problem discussed is that of determining the finger joint motion of the object. The last problem discussed is that of determining the ranges of motion of an object grasped by a hand.

Dai and Rees Jones ${ }^{8}$ gives a novel view of new metamorphic mechanisms and some development in deployable mechanisms and can be developed into multifingered robot hand, reconfigurable robot arms. The metamorphic mechanisms ${ }^{9}$ takes the concept of metamorphosis and changes its structure, shape and subsequently mobility ${ }^{10}$ to adapt to the environments and

Journal of Mechanical Engineering, vol. ME36, Dec. 2006

Transaction of the Mech. Eng. Div., The Institution of Engineers, Bangladesh. 
to meet the demands. Innovative design has been made in developing new robotic kinematics structure.

Dai and Rees Jones ${ }^{8,9}$ discusses about the characteristics of metamorphic mechanism, the facilities of changing the number of effective links and the change of configurations. Configuration changes from one form to another with a change in the mobility. They also discuss the different classes of mechanisms and focuses on the consequence of changing structures when erected or folded $^{10,}{ }^{11}$. Screw system theory is brought into consideration for the analysis of mechanisms with multiple loops. Different geometry and system combination are used to study the mobility and kinematics. McCarthy ${ }^{12}$ and Joseph duffy ${ }^{13}$ analyzed the spherical linkage mechanism. These linkages have the property that every link in the system rotates about the same fixed point. Thus trajectories of points in each link lie on concentric sphere with this point as centre. Only revolute joint is compatible with this rotational movement and its axis must pass through the fixed point. A spherical manipulator is essentially an open spherical polygon or unclosed loop with one end fixed to ground and a free end carrying mechanical finger or gripping device.

Mathematical Representation of Four Bar Spherical Linkage Mechanism:

The mathematical formulations are important to understand the spatial displacement of a four-bar spherical mechanism .In a spherical linkages mechanism every link in the system rotates about the same fixed point and the trajectories of points in each link lies on concentric sphere with this point as centre. The links have revolute joint in between them and the joint axis pass through the centre point. The link configurations are the functions of the joint variables and the dimensions of the links ${ }^{12,13}$.

A revolute joint in a spherical mechanism allows spatial rotation about its axis. To define this rotation, a fixed frame $\mathrm{F}$ at the centre and a moving frame $M$ have been introduced attached to the moving link as shown in the following figure. The coordinate transformation between these frames defines the rotation of the link.

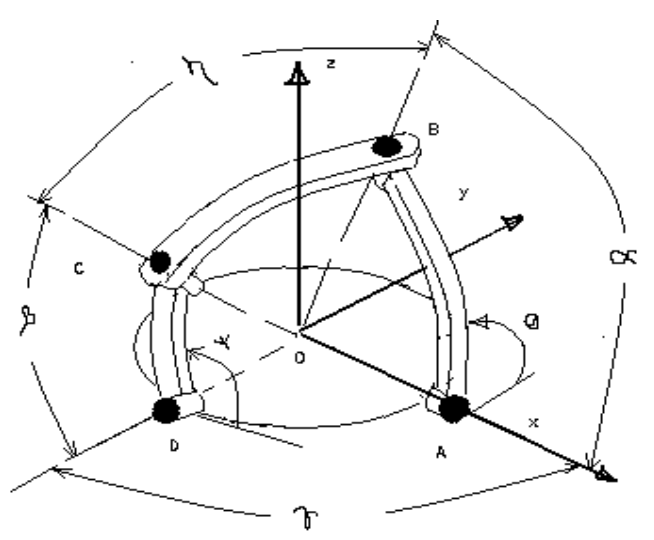

Coordinate rotation in a 4R spherical linkage (Spherical quadrilateral)

Consider a link connected to ground by one revolute joint. $\mathbf{A}$ is directed along the axis of this joint and $\mathbf{B}$ is defining the other end of the link. Both A \& B are unit vectors that at the centre $\mathbf{0}$. The angle, $\alpha$, between these vectors define the size of this link. The origin of fixed frame $\mathrm{F}(\mathrm{x}, \mathrm{y}, \mathrm{z})$ is at, $\mathbf{0}$ and its $\mathrm{x}$-axis is directed along $\mathbf{A}$ and its $\mathrm{y}$-axis is directed along $\mathbf{A x B}$. This convention ensures that $\mathbf{B}$ has $\sin \alpha$ at its positive $\mathrm{z}$ component. Attach the moving frame $M$ to $\mathbf{A B}$ so that in the initial configuration it is aligned with $\mathrm{F}$. As the crank rotates the angle $\theta$ measured counter clockwise about $\mathbf{A}$ from the $\mathrm{z}$ axis of $\mathrm{F}$ to the $\mathrm{z}$-axis of $M$ defines the rotation of the link ${ }^{12}$.

The orientation of $\mathbf{A B}$ is defined by transformation between coordinates

$\mathbf{x}=(x, y, z)^{T}$ in $M$ to $\mathbf{X}=(X, Y, Z)^{T}$ in $\mathrm{F}$, given by matrix equation

$$
\left[\begin{array}{l}
X \\
Y \\
Z
\end{array}\right]=\left[\begin{array}{ccc}
1 & 0 & 0 \\
0 & \cos \theta & -\sin \theta \\
0 & \sin \theta & \cos \theta
\end{array}\right]\left[\begin{array}{l}
x \\
y \\
z
\end{array}\right]
$$

Or $\quad \mathbf{X}=[\mathrm{X}(\theta)] \mathbf{x}$

The notation $[\mathrm{X}(\theta)]$ represents a rotation of angle $\theta$ about the X-axis. Similar matrices can be defined [Y $(\alpha)],[Z(\alpha)]$ to represent rotations about the $y$-axis and $z$ axis, given by

$Y(\alpha)]=\left[\begin{array}{ccc}\cos \alpha & 0 & \sin \alpha \\ 0 & 1 & 0 \\ -\sin \alpha & 0 & \cos \alpha\end{array}\right]$
$[Z(\alpha)]=\left[\begin{array}{ccc}\cos \alpha & -\sin \alpha & 0 \\ \sin \alpha & \cos \alpha & 0 \\ 0 & 0 & 1\end{array}\right]$

Let $\mathbf{b}=(\cos \alpha, 0, \sin \alpha)^{T}$ be the coordinates in $M$ of the vector $\mathbf{B}$ in the link $\mathbf{A B}$. b can be defined by rotating the unit vector $\underline{k}=(1,0,0)^{T}$ about the y-axis, so we have, $\mathbf{b}=[\mathrm{Y}(\alpha)] \underline{k}$. To obtain $\mathbf{B}$ after a rotation by $\theta$ which is given by the composition of two coordinate rotations ${ }^{12}$.

$\mathbf{B}=[\mathrm{X}(\theta)][\mathrm{Y}(\alpha)] \underline{k}$.

This equation can be read from right to left as the rotation of $\underline{k}$ by the angle $\alpha$ around $y$-axis followed by a rotation by $\theta$ around z-axis. The coordinates of $\mathbf{A}, \mathbf{D}, \mathbf{B}$ and $\mathbf{C}$ define by $\mathbf{A}=\underline{k} ; \mathbf{D}=[\mathrm{Z}(\gamma)] \underline{k} ; \mathbf{B}=[\mathrm{X}(\theta)][\mathrm{Y}(\alpha)] \underline{k}$ \& $\mathbf{C}=[\mathrm{Z}(\gamma)][\mathrm{X}(\psi)][\mathrm{Z}(\beta)] \underline{k}$

The angular lengths of each of the links of the spherical quadrilateral ABCD that defines a spherical $4 \mathrm{R}$ linkage, $\alpha=\operatorname{arcos}(\mathbf{A}$. B $), \beta=\operatorname{arcos}(\mathbf{C}$. D), $\gamma=\operatorname{arcos}$ ( D . A) and $\eta=\operatorname{arcos}(\mathbf{B}$. C)

These dimensions completely define the movement of the linkage ${ }^{12}$.

This presented the position analysis of spherical fourbar closed chain mechanism and the mathematical formulations of the design equations. The kinematic and geometrical theory of spatial rotation has fundamental importance in analysing and developing mathematical model of spherical mechanism. 
Geometrical and Mathematical Design of five Bar Spherical Linkage Metamorphic Robot Palm:

A generalised geometric model of a five bar spherical metamorphic mechanism is shown in the figure 1. Point o is considered as the centre of the sphere. Position 1, 2, 3, 4 $\& 5$ are the revolute joints of the spherical linkages. o 1 , o 2 , o 3 , o 4 and o 5 represents the concentric axis of the revolute joints. $\mathrm{f} 1, \mathrm{f} 2, \mathrm{f} 3$ are the three finger position in the spherical link $\mathrm{L}_{12}, \mathrm{~L}_{45} \& \mathrm{~L}_{51}$. Link $\mathrm{L}_{51}$ is considered as reference or fixed link.

The angle subtended by the links $\mathrm{L}_{12}, \mathrm{~L}_{23}, \mathrm{~L}_{34}, \mathrm{~L}_{45}$, $\mathrm{L}_{51}$ are respectively $\alpha, \beta, \gamma, \delta, \eta$. $\rho_{1 \mathrm{f}}$ represents the angle created in the centre by the axis of the revolute joint 1 and the finger position $\mathrm{fl}$. Similarly $\rho_{4 \mathrm{f}}$ is the angle subtended by the joint axis 4 and the finger position $\mathrm{f} 2$ and $\rho_{5 \mathrm{f}}$ is the angle between joint axis 5 and finger position f3. The mechanism structure and its capability of achieving a good range of configurations will depend on the selection of the angles $\alpha, \beta, \gamma, \delta, \eta, \rho_{1 \mathrm{f}}, \rho_{4 \mathrm{f}}, \rho_{5 \mathrm{f}}$. Linear distance between the joints of each link $\mathrm{L}_{12}, \mathrm{~L}_{23}, \mathrm{~L}_{34}, \mathrm{~L}_{45}, \mathrm{~L}_{51}$ termed as $l_{1}, l_{2}$, $l_{3}, l_{4}$ and $l_{5}$. These linear distances will be used later on for developing the transformation matrices.

A fixed co-ordinate frame $\mathrm{F}\left(\mathrm{X}_{\mathrm{o}}, \mathrm{Y}_{\mathrm{o}}, \mathrm{Z}_{\mathrm{o}}\right)$ has been considered at the centre of the sphere. The origin of the fixed co-ordinate frame $\mathrm{F}$ is $\mathrm{o}$, which is also the centre of the sphere. The $\mathrm{x}$-axis has been placed along the joint axis
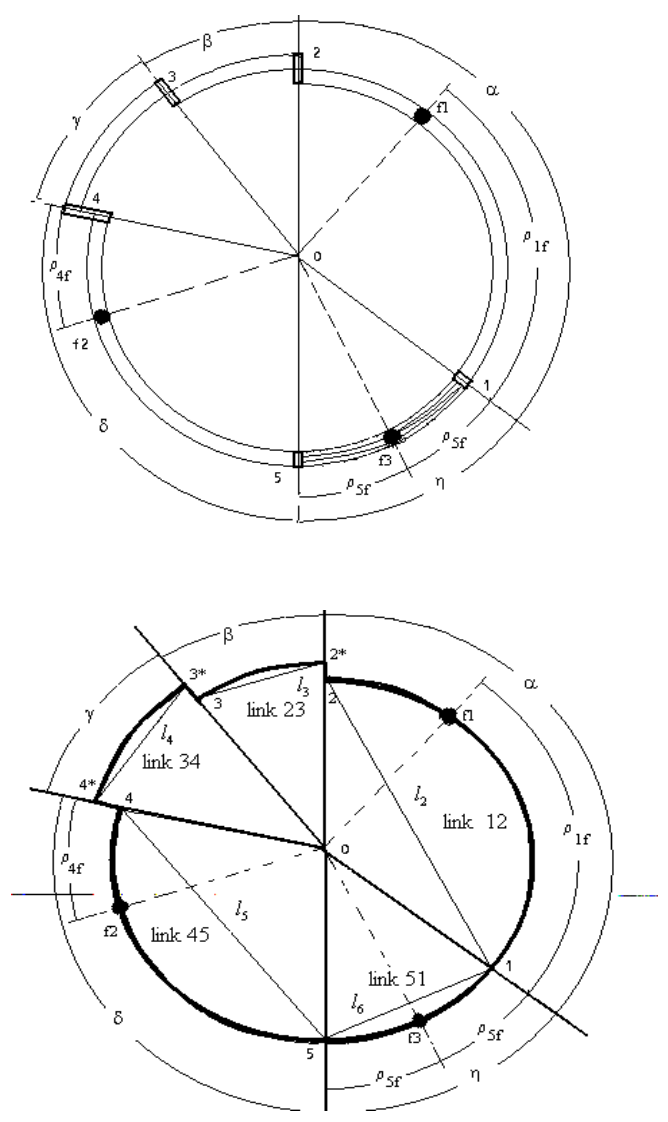

Figure1: Geometric configuration of five bar spherical linkage metamorphic mechanism of joint 1 . This set up of the co-ordinate frame is shown in the Figure 2. To define the coordinate rotation and transformation, moving frames are attached to the position $1, \mathrm{f1}, 2,3,4, \mathrm{f} 2,5$ and $\mathrm{f} 3$. Now through the development of the transformation matrices the kinematics of the links can be described.

The transformation matrix of the fixed frame $\mathrm{F}\left(\mathrm{X}_{\mathrm{o}}\right.$, $\mathrm{Y}_{\mathrm{o}}, \mathrm{Z}_{\mathrm{o}}$ ) at the origin is

$$
\mathrm{T}_{0}^{0}=\left[\begin{array}{llll}
1 & 0 & 0 & 0 \\
0 & 1 & 0 & 0 \\
0 & 0 & 1 & 0 \\
0 & 0 & 0 & 1
\end{array}\right]
$$

Which is the form of homogenous transformation matrix

$$
\left[\begin{array}{llll}
(R) & & & (d) \\
0 & 0 & 0 & 1
\end{array}\right]
$$

Where (R) is the $3 \times 3$ orientation matrix and (d) is the 3 $\mathrm{x} 1$ position matrix. The position matrix will define the $\mathrm{x}$, $\mathrm{y}, \mathrm{z}$ co-ordinate of that particular point in the three dimensional space. The coordinate transformation matrix of the joint position 1 relative to the fixed frame $F\left(X_{0}, Y_{0}\right.$, $\mathrm{Z}_{\mathrm{o}}$ ) will be denoted $\mathrm{asT}_{0}{ }^{1}$. The relative coordinate transformation matrix at different joint and finger position will be derived.
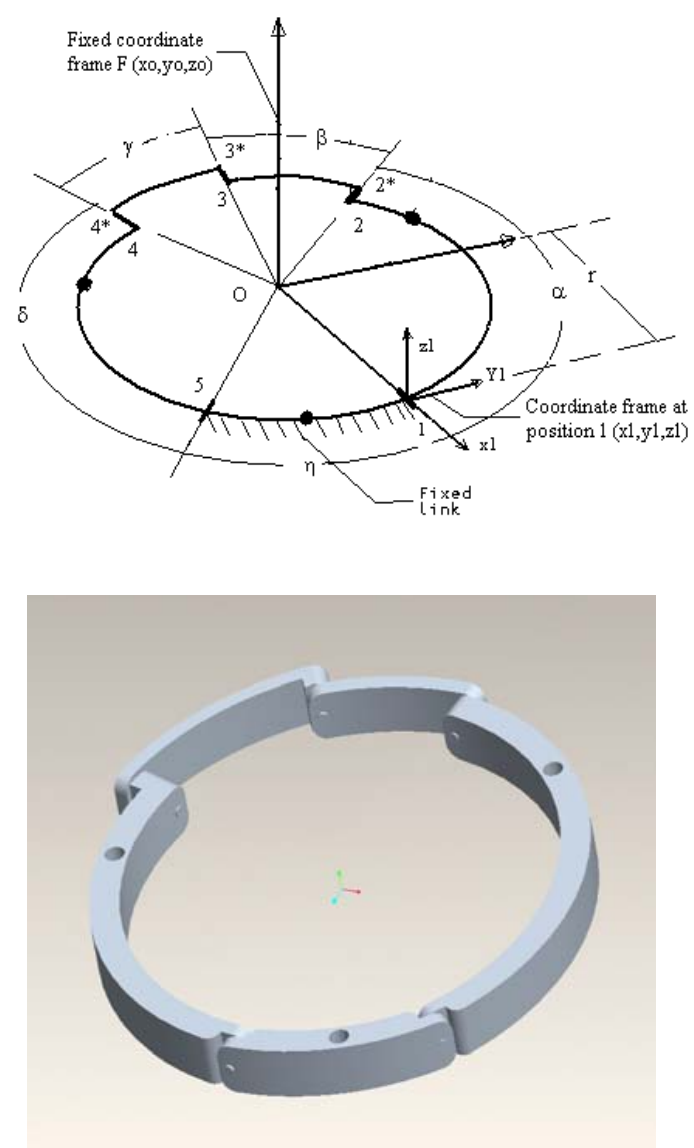

Figure 2: Coordinate transformation at joint position 1 
Coordinate transformation matrix at joint axis position 1

To define the transformation matrix both the translation and the rotation of the moving frame have to be considered. Figure 2 shows the co-ordinate frame at joint position 1. To derive the transformation matrix at joint position 1 with respect to fixed frame $F$, translation of $r$ along the $\mathrm{x}$-axis with respect to fixed frame has been considered. Where $r$ is the radius of the circle. But there is no rotation of the co-ordinate frame with respect to the fixed frame.

$\mathrm{T}_{0}{ }^{1}=$ Translation of $(\mathrm{r}, 0,0,1)$ and no rotation with respect to fixed frame $\mathrm{F}$.

$\mathrm{T}_{0}{ }^{1}=\left[\begin{array}{cccc}1 & 0 & 0 & r \\ 0 & 1 & 0 & 0 \\ 0 & 0 & 1 & 0 \\ 0 & 0 & 0 & 1\end{array}\right]$ and $\left[\begin{array}{c}X_{1} \\ Y_{1} \\ Z_{1}\end{array}\right]=\left[\begin{array}{c}r \\ 0 \\ 0\end{array}\right]$

Where $\mathrm{T}_{0}{ }^{1}$ represents the transformation matrix of joint axis point 1 with respect to the fixed frame $F$ and $X_{1}, Y_{1}, Z_{1}$ represents the $\mathrm{x}, \mathrm{y}, \mathrm{z}$ co-ordinate in the space with respect to fixed frame.

\section{Coordinate transformation matrix of finger position $\mathrm{f} 1$}

The link configuration changes as the link $\mathrm{L}_{12}$ start to rotate about the joint axis 1 . Figure 3 shows the angular displacement $\theta_{1}$ of the link $\mathrm{L}_{12}$ with respect to the reference link $\mathrm{L}_{51}$. The point $\mathrm{fl}$ lies on the moving link $\mathrm{L}_{12}$.

To define the transformation matrix at finger position $\mathrm{fl}$, a translation along the $\mathrm{x}, \mathrm{y}$ and $\mathrm{z}$-axis and a rotation about the $\mathrm{x}$ and $\mathrm{z}$ axis with respect to frame at position 1 has to be considered. Figure 4 shows the geometric configuration to determine the translation in the $\mathrm{x}, \mathrm{y}$ and $\mathrm{z}$-axis with respect to co-ordinate at point 1 .

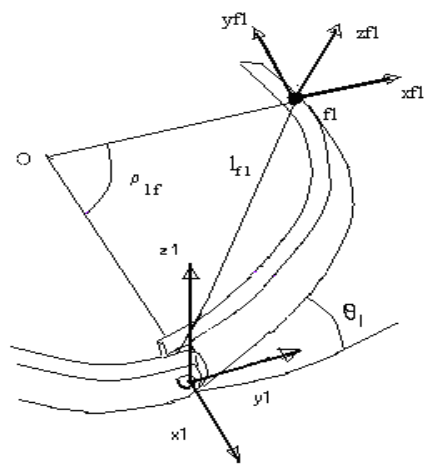

Figure 3: Relative angular rotation of the link

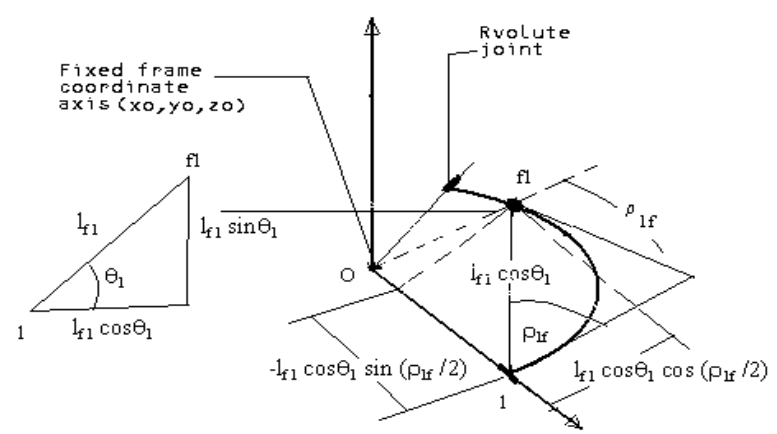

Figure 4: Translation of position $\mathrm{fl}$ relative to position 1
The translation along the $\mathrm{x}$-axis is $-l_{\mathrm{f} 1} \cos \theta_{1} \sin \left(\rho_{1 \mathrm{f}}\right.$ $/ 2)$, along the $y$-axis is $l_{\mathrm{f} 1} \cos \theta_{1} \cos \left(\rho_{1 \mathrm{f}} / 2\right)$ and along the $\mathrm{z}$ axis is $l_{\mathrm{fl}} \sin \theta_{1}$ with respect to co-ordinate at point 1 .

The transformation matrix of point $\mathrm{fl}$ with respect to point 1 termed as $\mathrm{T}_{1}{ }^{\mathrm{fl}}$ can be defined by the composition of translation along $\mathrm{x}, \mathrm{y}$ and $\mathrm{z}$ axis and rotation of $\theta_{1}$ about $\mathrm{x}$ axis and $\rho_{1 \mathrm{f}}$ about $\mathrm{z}$-axis.

$\mathrm{T}_{1}{ }^{\mathrm{fl}}=\left[\begin{array}{cccc}C \rho_{1 f} & -S \rho_{1 f} & 0 & -l_{f_{1}} C \theta_{1} S\left(\rho_{1 f} / 2\right) \\ S \rho_{1 f} C \theta_{1} & C \rho_{1 f} C \theta_{1} & -S \theta_{1} & l_{f_{1}} C \theta_{1} C\left(\rho_{1 f} / 2\right) \\ S \rho_{1 f} S \theta_{1} & C \rho_{1 f} S \theta_{1} & C \theta_{1} & l_{f_{1}} S \theta_{1} \\ 0 & 0 & 0 & 1\end{array}\right]$

Where $\sin =\mathrm{S}$ and $\cos =\mathrm{C}$

By applying the composition law of transformation matrices we can derive the co-ordinate transformation matrix $\mathrm{T}_{0}{ }^{\mathrm{fl}}$ of point $\mathrm{fl}$ with respect to the (origin) fixed frame $\mathrm{F}$.

$$
\begin{aligned}
& \mathrm{T}_{0}{ }^{\mathrm{f} 1}=\mathrm{T}_{0}{ }^{1} \mathrm{~T}_{1}{ }^{\mathrm{f} 1} \\
& = \\
& {\left[\begin{array}{llll}
1 & 0 & 0 & r \\
0 & 1 & 0 & 0 \\
0 & 0 & 1 & 0 \\
0 & 0 & 0 & 1
\end{array}\right]\left[\begin{array}{cccc}
C \rho_{1 f} & -S \rho_{1 f} & 0 & -l_{f_{1}} C \theta_{1} S\left(\rho_{1 f} / 2\right) \\
S \rho_{1 f} C \theta_{1} & C \rho_{1 f} C \theta_{1} & -S \theta_{1} & l_{f_{1}} C \theta_{1} C\left(\rho_{1 f} / 2\right) \\
S \rho_{1 f} S \theta_{1} & C \rho_{1 f} S \theta_{1} & C \theta_{1} & l_{f_{1}} S \theta_{1} \\
0 & 0 & 0 & 1
\end{array}\right]} \\
& =\left[\begin{array}{cccc}
C \rho_{1 f} & -S \rho_{1 f} & 0 & -l_{f_{1}} C \theta_{1} S\left(\rho_{1 f} / 2\right)+r \\
S \rho_{1 f} C \theta_{1} & C \rho_{1 f} C \theta_{1} & -S \theta_{1} & l_{f_{1}} C \theta_{1} C\left(\rho_{1 f} / 2\right) \\
S \rho_{1 f} S \theta_{1} & C \rho_{1 f} S \theta_{1} & C \theta_{1} & l_{f_{1}} S \theta_{1} \\
0 & 0 & 0 & 1
\end{array}\right]
\end{aligned}
$$

From the transformation matrix the position of the point $\mathrm{fl}$ with respect to the fixed frame can be derived

$$
\left[\begin{array}{c}
X_{f_{l}} \\
Y_{f_{l}} \\
Z_{f_{l}}
\end{array}\right]=\left[\begin{array}{c}
-l_{f_{1}} C \theta_{1} S\left(\rho_{1 f} / 2\right)+r \\
l_{f_{1}} C \theta_{1} C\left(\rho_{1 f} / 2\right) \\
l_{f_{1}} S \theta_{1}
\end{array}\right]
$$

Where $\mathrm{X}_{\mathrm{fl}}, \mathrm{Y}_{\mathrm{fl}}, \mathrm{Z}_{\mathrm{fl}}$ are the coordinate positions in the three dimensional space. As the value $l_{\mathrm{f} 1}$ and $\rho_{1 \mathrm{f}}$ can be fixed according to geometric design parameter,

$$
\mathrm{X}_{\mathrm{fl}}=f\left(\theta_{l}\right), \mathrm{Y}_{\mathrm{fl}}=f\left(\theta_{l}\right) \text { and } \mathrm{Z}_{\mathrm{fl}}=f\left(\theta_{l}\right)
$$

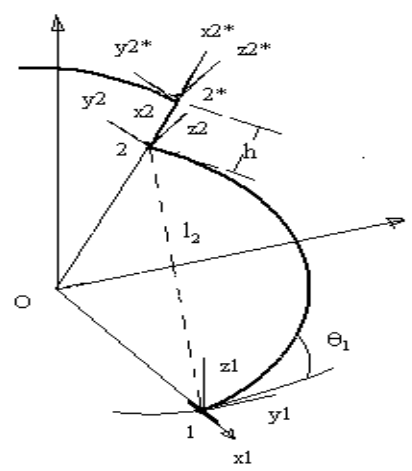

Figure 5: Coordinate transformation at position 2

Journal of Mechanical Engineering, vol. ME36, Dec. 2006

Transaction of the Mech. Eng. Div., The Institution of Engineers, Bangladesh. 
Coordinate transformation matrix of position 2 and 2 *

The coordinate transformation matrix $\mathrm{T}_{1}^{2}$ of joint position 2 with respect to joint position 1 can be derived in the similar manner as described earlier. Figure 5 shows the transformed co-ordinate at point 2 and $2 *$ with respect to point 1 when link $L_{12}$ has an angular displacement of $\theta_{1}$ relative to link $\mathrm{L}_{51}$. The translation to be considered along $\mathrm{x}$-axis is $l_{2} \mathrm{C} \theta_{1} \mathrm{~S}(\alpha / 2)$, along $\mathrm{y}$-axis $l_{2} \mathrm{C} \theta_{1} \mathrm{C}(\alpha / 2)$, and along $\mathrm{z}$-axis $l_{2} \mathrm{~S} \theta_{1}$ followed by the rotation of $\theta_{1}$ about $\mathrm{x}$-axis and $\alpha$ about $z$-axis.

$\mathrm{T}_{1}^{2}=\left[\begin{array}{cccc}C \alpha & -S \alpha & 0 & -l_{2} C \theta_{1} S(\alpha / 2) \\ S \alpha C \theta_{1} & C \alpha C \theta_{1} & -S \theta_{1} & l_{2} C \theta_{1} C(\alpha / 2) \\ S \alpha S \theta_{1} & C \alpha S \theta_{1} & C \theta_{1} & l_{2} S \theta_{1} \\ 0 & 0 & 0 & 1\end{array}\right]$

$\mathrm{T}_{0}^{2}=\mathrm{T}_{0}^{1} \mathrm{~T}_{1}^{2}=$

$\left[\begin{array}{cccc}C \alpha & -S \alpha & 0 & -l_{2} C \theta_{1} S(\alpha / 2)+r \\ S \alpha C \theta_{1} & C \alpha C \theta_{1} & -S \theta_{1} & l_{2} C \theta_{1} C(\alpha / 2) \\ S \alpha S \theta_{1} & C \alpha S \theta_{1} & C \theta_{1} & l_{2} S \theta_{1} \\ 0 & 0 & 0 & 1\end{array}\right]$ and

$\left[\begin{array}{c}X_{2} \\ Y_{2} \\ Z_{2}\end{array}\right]=\left[\begin{array}{c}-l_{2} C \theta_{1} S(\alpha / 2)+r \\ l_{2} C \theta_{1} C(\alpha / 2) \\ l_{2} S \theta_{1}\end{array}\right]$

Where $\mathrm{X}_{2}, \mathrm{Y}_{2}, \mathrm{Z}_{2}$ are the coordinate positions in the three dimensional space. As the value $l_{2}$ and $\alpha$ can be set according to geometric design parameter, $\mathrm{X}_{2}=f\left(\theta_{1}\right), Y_{2}=f$ $\left(\theta_{I}\right)$ and $\mathrm{Z}_{2}=f\left(\theta_{I}\right)$

The point 2 and $2 *$ are on the same axis but of two different link. Point 2 is described as the joint position of link $\mathrm{L}_{12}$ and point $2 *$ is the joint position of link $\mathrm{L}_{23}$ on the same joint axis as shown in the figure 4.8. The co-ordinate orientation matrix of these two points will same but there position matrix will differ as, $h$ is the distance between the two points. The transformation matrix $\mathrm{T}_{2}{ }^{2 *}$ of point $2^{*}$ with respect two point 2 , can be derived by considering only translation of $\mathrm{h}$ along $\mathrm{x}$-axis and no rotation.

$\mathrm{T}_{2}^{2 *}=\left[\begin{array}{cccc}1 & 0 & 0 & h \\ 0 & 1 & 0 & 0 \\ 0 & 0 & 1 & 0 \\ 0 & 0 & 0 & 1\end{array}\right]$ and

$\mathrm{T}_{0}^{2 *}=\mathrm{T}_{0}{ }^{2} \mathrm{~T}_{2} 2^{*}\left[\begin{array}{cccc}C \alpha & -S \alpha & 0 & h C \alpha-l_{2} C \theta_{1} S(\alpha / 2)+r \\ S \alpha C \theta_{1} & C \alpha C \theta_{1} & -S \theta_{1} & h S \alpha C \theta_{1}+l_{2} C \theta_{1} C(\alpha / 2) \\ S \alpha S \theta_{1} & C \alpha S \theta_{1} & C \theta_{1} & h S \alpha S \theta_{1}+l_{2} S \theta_{1} \\ 0 & 0 & 0 & 1\end{array}\right]$

From the above transformation matrix the position of the point $2^{*}$ in the space with respect to the fixed frame can be derived as $\left[\begin{array}{c}X_{2^{*}} \\ Y_{2^{*}} \\ Z_{2^{*}}\end{array}\right]=\left[\begin{array}{c}h C \alpha-l_{2} C \theta_{1} S(\alpha / 2)+r \\ h S \alpha C \theta_{1}+l_{2} C \theta_{1} C(\alpha / 2) \\ h S \alpha S \theta_{1}+l_{2} S \theta_{1}\end{array}\right]$

where $\mathrm{h}$ is the linear distance between the point 2 and $2^{*}$.As the value $l_{2}, \mathrm{~h}$ and $\alpha$ can be set according to geometric design parameter, $\mathrm{X}_{2^{*}}=f\left(\theta_{l}\right), \mathrm{Y}_{2^{*}}=f\left(\theta_{l}\right)$ and $\mathrm{Z}_{2^{*}}=f\left(\theta_{1}\right)$

Coordinate transformation matrix of position $3 \& 3 *$

According to the mechanism displacement link $\mathrm{L}_{23}$ will have an angular displacement of $\theta_{2}$ relative to link $\mathrm{L}_{12}$. The coordinate transformation matrix $\mathrm{T}_{2 *}{ }^{3}$ of joint position 3 with respect to joint position $2^{*}$, can be derived by considering both translation and rotation of the coordinate axis. The translation to be considered along $\mathrm{x}$-axis is $-l_{3} \mathrm{C}_{2} \mathrm{~S}(\beta / 2)$, along $\mathrm{y}$-axis $l_{3} \mathrm{C} \theta_{2} \mathrm{C}(\beta / 2)$, and along $\mathrm{z}$ axis $l_{3} \mathrm{~S} \theta_{2}$, followed by the rotation of $\theta_{2}$ about $\mathrm{x}$-axis and $\beta$ about $\mathrm{z}$-axis.

$$
\begin{aligned}
& \mathrm{T}_{0}{ }^{3}=\mathrm{T}_{0}{ }^{2 *} \mathrm{~T}_{2^{*}}{ }^{3}=\left[\begin{array}{llll}
m_{11} & m_{12} & m_{13} & m_{14} \\
m_{21} & m_{22} & m_{23} & m_{24} \\
m_{31} & m_{32} & m_{33} & m_{34} \\
m_{41} & m_{42} & m_{43} & m_{44}
\end{array}\right] \\
& \text { and }\left[\begin{array}{l}
X_{3} \\
Y_{3} \\
Z_{3}
\end{array}\right]=\left[\begin{array}{l}
m_{14} \\
m_{24} \\
m_{34}
\end{array}\right]
\end{aligned}
$$

$$
\begin{aligned}
& m_{11}=C \alpha C \beta-S \alpha S \beta C \theta_{2} ; \\
& m_{12}=-C \alpha S \beta-S \alpha C \beta C \theta_{2} ; m_{13}=-S \alpha S \theta_{2} \\
& m_{14}=-l_{3} C \theta_{2} S(\beta / 2) C \alpha-l_{3} C \theta_{2} C(\beta / 2) S \alpha+ \\
& \quad h C \alpha-l_{2} C \theta_{1} S(\alpha / 2)+r \\
& m_{21}=S \alpha C \theta_{1} C \beta+C \alpha C \theta_{1} S \beta C \theta_{2}-S \theta_{1} S \beta S \theta_{2} ; \\
& m_{22}=-S \beta S \alpha C \theta_{1}+C \alpha C \theta_{1} C \beta C \theta_{2}-C \beta S \theta_{2} S \theta_{1} \\
& m_{23}=-C \beta S \theta_{2} S \theta_{1}-S \theta_{1} C \theta_{2} ; \\
& m_{24}=-l_{3} C \theta_{2} S(\beta / 2) S \alpha C \theta_{1}+ \\
& \quad l_{3} C \theta_{2} C(\beta / 2) C \alpha C \theta_{1}- \\
& \quad l_{3} S \theta_{2} S \theta_{1}+h S \alpha C \theta_{1}+l_{2} C \theta_{1} C(\alpha / 2) \\
& m_{31}=S \alpha S \theta_{1} C \beta+C \alpha S \theta_{1} S \beta C \theta_{2}+C \theta_{1} S \beta S \theta_{2} ; \\
& m_{32}=-S \beta S \alpha_{1} S \theta_{1}+C \alpha S \theta_{1} C \beta C \theta_{2}+C \theta_{1} C \beta S \theta_{2} ; \\
& m_{33}=-S \theta_{2} C \alpha S \theta_{1}+C \theta_{1} C \theta_{2} ; \\
& m_{34}=-l_{3} C \theta_{2} S\left(\frac{\beta}{2}\right) S \alpha S \theta_{1}+l_{3} C \theta_{2} C\left(\frac{\beta}{2}\right) C \alpha S \theta_{1} \\
& \quad+l_{3} S \theta_{2} C \theta_{1}+h S \alpha S \theta_{1}+l_{2} S \theta_{1} \\
& m_{41}=0 ; \quad m_{42}=0 ; m_{43}=0 ; m_{44}=1
\end{aligned}
$$

As the value $l_{3}, \mathrm{~h}, \mathrm{r}, \alpha$ and $\beta$ can be fixed value according to geometric design parameter, $\mathrm{X}_{3}=f\left(\theta_{l}, \theta_{2}\right)$, $\mathrm{Y}_{3}=f\left(\theta_{1}, \theta_{2}\right)$ and $\mathrm{Z}_{3}=f\left(\theta_{1}, \theta_{2}\right)$

The point 3 and $3 *$ are on the same axis but of two different link. Point 3 is described as the joint position of link $\mathrm{L}_{23}$ and point $3 *$ is the joint position of link $\mathrm{L}_{34}$ on the same joint axis. The co-ordinate orientation matrix of these two points will be same but the position matrix will differ, as $\mathrm{h}$ is the distance between the two points. 


$$
\begin{gathered}
\mathrm{T}_{0}{ }^{* *}=\mathrm{T}_{0}{ }^{3} \mathrm{~T}^{3 *}=\left[\begin{array}{llll}
n_{11} & n_{12} & n_{13} & n_{14} \\
n_{21} & n_{22} & n_{23} & n_{24} \\
n_{31} & n_{32} & n_{33} & n_{34} \\
n_{41} & n_{42} & n_{43} & n_{44}
\end{array}\right] \text { and } \\
\text { coordinates }\left[\begin{array}{c}
X_{3^{*}} \\
Y_{3^{*}} \\
Z_{3^{*}}
\end{array}\right]=\left[\begin{array}{l}
n_{14} \\
n_{24} \\
n_{34}
\end{array}\right]
\end{gathered}
$$

where

$$
\begin{gathered}
n_{14}=-l_{3} C \theta_{2} S(\beta / 2) C \alpha-l_{3} C \theta_{2} C(\beta / 2) S \alpha+ \\
h C \alpha-l_{2} C \theta_{1} S(\alpha / 2)+h\left(C \alpha C \beta-S \alpha S \beta C \theta_{2}\right)+r \\
n_{24}=-l_{3} C \theta_{2} S(\beta / 2) S \alpha C \theta_{1}+l_{3} C \theta_{2} C(\beta / 2) C \alpha C \theta_{1}- \\
l_{3} S \theta_{2} S \theta_{1}+h S \alpha C \theta_{1}+l_{2} C \theta_{1} C(\alpha / 2)+h\left(S \alpha C \theta_{1} C \beta+\right. \\
\left.C \alpha C \theta_{1} S \beta C \theta_{2}-S \theta_{1} S \beta S \theta_{2}\right) \\
n_{34}=-l_{3} C \theta_{2} S(\beta / 2) S \alpha S \theta_{1}+ \\
l_{3} C \theta_{2} C(\beta / 2) C \alpha S \theta_{1}+l_{3} S \theta_{2} C \theta_{1}+ \\
h S \alpha S \theta_{1}+l_{2} S \theta_{1}+h\left(S \alpha S \theta_{1} C \beta+\right. \\
\left.C \alpha S \theta_{1} S \beta C \theta_{2}+C \theta_{1} S \beta S \theta_{2}\right)
\end{gathered}
$$

As the value $l_{3}, \mathrm{~h}, \mathrm{r}, \alpha$ and $\beta$ will be fixed value according to geometric design parameter, $\mathrm{X}_{3^{*}}=f\left(\theta_{1}, \theta_{2}\right), Y 3_{*}=f$ $\left(\theta_{1}, \theta_{2}\right)$ and $\mathrm{Z}_{3^{*}}=f\left(\theta_{1}, \theta_{2}\right)$

\section{Coordinate transformation matrix of position $4 * \& 4$}

Link $L_{34}$ will have an angular displacement of $\theta_{3}$ relative to link $\mathrm{L}_{23}$. The coordinate transformation matrix $\mathrm{T}_{3^{*}}{ }^{*}$ of joint position $4 *$ with respect to joint position $3 *$, can be derived by considering both translation and rotation of the co-ordinate axis .The translation to be considered along $\mathrm{x}$-axis is $-l_{4} \mathrm{C}_{3} \mathrm{~S}(\gamma / 2)$, along $\mathrm{y}$-axis $l_{4} \mathrm{C} \theta_{3} \mathrm{C}(\gamma / 2)$, and along z-axis $l_{4} \mathrm{~S}_{3}$, followed by the rotation of $\theta_{3}$ about $\mathrm{x}$ axis and $\gamma$ about $\mathrm{z}$-axis. The co-ordinates of point $4^{*}$ with respect to the (origin) fixed frame $F$,

$$
\begin{aligned}
& \mathrm{T}_{\mathrm{o}}{ }^{4^{*}}=\mathrm{T}_{\mathrm{o}}{ }^{3 *} \mathrm{~T}_{3^{*}}{ }^{4^{*}}=\left[\begin{array}{llll}
p_{11} & p_{12} & p_{13} & p_{14} \\
p_{21} & p_{22} & p_{23} & p_{24} \\
p_{31} & p_{32} & p_{33} & p_{34} \\
p_{41} & p_{42} & p_{43} & p_{44}
\end{array}\right] \text { and } \\
& {\left[\begin{array}{c}
\mathrm{X}_{4^{*}} \\
\mathrm{Y}_{4^{*}} \\
\mathrm{Z}_{4^{*}}
\end{array}\right]=\left[\begin{array}{l}
p_{14} \\
p_{24} \\
p_{34}
\end{array}\right]}
\end{aligned}
$$

where

$$
\begin{aligned}
& p_{14}=-n_{11} l_{4} C \theta_{3} S(\gamma / 2)+n_{12} l_{4} C \theta_{3} C(\gamma / 2)+n_{13} \\
& l_{4} S \theta_{3}+n_{14} \\
& p_{24}=-n_{21} l_{4} C \theta_{3} S(\gamma / 2)+n_{22} l_{4} C \theta_{3} C(\gamma / 2)+n_{23} \\
& l_{4} S \theta_{3}+n_{24} \\
& p_{34}=-n_{31} l_{4} C \theta_{3} S(\gamma / 2)+n_{32} l_{4} C \theta_{3} C(\gamma / 2)+n_{33} \\
& l_{4} S \theta_{3}+n_{34}
\end{aligned}
$$

As the value $l_{1}, l_{2}, l_{3}, l_{4}, \mathrm{~h}, \mathrm{r}, \alpha, \beta$ and $\gamma$ will be fixed value according to geometric design parameter, $\mathrm{X}_{4^{*}}=f\left(\theta_{1}, \theta_{2}, \theta_{3}\right)$, $\mathrm{Y}_{4^{*}}=f\left(\theta_{1}, \theta_{2}, \theta_{3}\right)$ and $\mathrm{Z}_{4^{*}}=f\left(\theta_{1}, \theta_{2}, \theta_{3}\right)$

$\mathrm{T}_{0}^{4}=\mathrm{T}_{0}{ }^{4 *} \mathrm{~T}_{4 *}{ }^{4}=\left[\begin{array}{llll}q_{11} & q_{12} & q_{13} & q_{14} \\ q_{21} & q_{22} & q_{23} & q_{24} \\ q_{31} & q_{32} & q_{33} & q_{34} \\ q_{41} & q_{42} & q_{43} & q_{44}\end{array}\right]$ and

$$
\left[\begin{array}{c}
X_{4} \\
Y_{4} \\
Z_{4}
\end{array}\right]=\left[\begin{array}{l}
q_{14} \\
q_{24} \\
q_{34}
\end{array}\right]=\left[\begin{array}{l}
f\left(\theta_{1} \theta_{2} \theta_{3}\right) \\
f\left(\theta_{1} \theta_{2} \theta_{3}\right) \\
f\left(\theta_{1} \theta_{2} \theta_{3}\right)
\end{array}\right]
$$

\section{Coordinate transformation matrix of finger position $f 2$ and point 5}

The point $\mathrm{f} 2 \& 5$ is situated on the link $\mathrm{L}_{45}$. With the change of the mechanism configuration link $\mathrm{L}_{45}$ will have an angular displacement of $\theta_{4}$ relative to link $\mathrm{L}_{34}$. The coordinate transformation matrix $\mathrm{T}_{4}{ }^{\mathrm{f} 2} \& \mathrm{~T}_{4}{ }^{5}$ can be derived by considering both translation and rotation of the coordinate axis. The translation to be considered along $\mathrm{x}$-axis is $-l_{\mathrm{f} 4} \mathrm{C} \theta_{4} \mathrm{~S}\left(\rho_{4 \mathrm{f}} / 2\right)$, , along y-axis $l_{\mathrm{f} 4} \mathrm{C} \theta_{4} \mathrm{~S}\left(\rho_{4 \mathrm{f}} / 2\right)$, and along z-axis $l_{\mathrm{f} 4} \mathrm{~S} \theta_{4}$, followed by the rotation of $\theta_{4}$ about $\mathrm{x}$-axis and $\rho_{4 \mathrm{f}}$ about z-axis.

$$
\begin{aligned}
& \mathrm{T}_{0}^{\mathrm{f} 2}=\mathrm{T}_{0}^{4} \mathrm{~T}_{4}^{\mathrm{f} 2}=\left[\begin{array}{llll}
s_{11} & s_{12} & s_{13} & s_{14} \\
s_{21} & s_{22} & s_{23} & s_{24} \\
s_{31} & s_{32} & s_{33} & s_{34} \\
s_{41} & s_{42} & s_{43} & s_{44}
\end{array}\right] \text { and } \\
& {\left[\begin{array}{c}
X_{f 2} \\
Y_{f 2} \\
Z_{f 2}
\end{array}\right]=\left[\begin{array}{l}
s_{14} \\
s_{24} \\
s_{34}
\end{array}\right]\left[\begin{array}{l}
f\left(\theta_{1} \theta_{2} \theta_{3} \theta_{4}\right) \\
f\left(\theta_{1} \theta_{2} \theta_{3} \theta_{4}\right) \\
f\left(\theta_{1} \theta_{2} \theta_{3} \theta_{4}\right)
\end{array}\right]}
\end{aligned}
$$

where

$$
\begin{gathered}
s_{14}=-q_{11} l_{f 4} C \theta_{4} S\left(\frac{\rho_{4 f}}{2}\right)+q_{12} l_{f 4} C \theta_{4} C\left(\frac{\rho_{4 f}}{2}\right)+ \\
q_{13} l_{f 4} S \theta_{4}+q_{14} \\
s_{24}=-q_{21} l_{f 4} C \theta_{4} S\left(\frac{\rho_{4 f}}{2}\right)+q_{22} l_{f 4} C \theta_{4} C\left(\frac{\rho_{4 f}}{2}\right)+ \\
q_{23} l_{f 4} S \theta_{4}+q_{14} \\
s_{34}=-q_{31} l_{f 4} C \theta_{4} S\left(\rho_{4 f} / 2\right)+q_{32} l_{f 4} C \theta_{4} C\left(\rho_{4 f} / 2\right)+ \\
q_{33} l_{f 4} S \theta_{4}+q_{34} \\
\mathrm{~T}_{0}{ }^{5}=\mathrm{T}_{0}{ }^{4} \mathrm{~T}_{4}{ }^{5}=\left[\begin{array}{llll}
t_{11} & t_{12} & t_{13} & t_{14} \\
t_{21} & t_{22} & t_{23} & t_{24} \\
t_{31} & t_{32} & t_{33} & t_{34} \\
t_{41} & t_{42} & t_{43} & t_{44}
\end{array}\right]
\end{gathered}
$$


and $\left[\begin{array}{c}X_{5} \\ Y_{5} \\ Z_{5}\end{array}\right]=\left[\begin{array}{c}t_{14} \\ t_{24} \\ t_{34}\end{array}\right]=\left[\begin{array}{l}f\left(\theta_{1} \theta_{2} \theta_{3} \theta_{4}\right) \\ f\left(\theta_{1} \theta_{2} \theta_{3} \theta_{4}\right) \\ f\left(\theta_{1} \theta_{2} \theta_{3} \theta_{4}\right)\end{array}\right]$

where

$$
\begin{gathered}
t_{14}=-q_{11} l_{5} C \theta_{4} S(\delta / 2)+q_{12} l_{5} C \theta_{4} C(\delta / 2) \\
+q_{13} l_{5} S \theta_{4}+q_{14} \\
t_{24}=-q_{21} l_{5} C \theta_{4} S(\delta / 2)+q_{22} l_{5} C \theta_{4} C(\delta / 2) \\
+q_{23} l_{5} S \theta_{4}+q_{24} \\
t_{34}=-q_{31} l_{5} C \theta_{4} S(\delta / 2)+q_{32} l_{5} C \theta_{4} C(\delta / 2) \\
\quad+q_{33} l_{5} S \theta_{4}+q_{34} \\
t_{41}=q_{41} C \delta+q_{42} S \delta C \theta_{4}+q_{43} S \delta S \theta_{4}
\end{gathered}
$$

\section{Coordinate transformation matrix of position 1 finger position $f_{3}$}

With the change of mechanism's configuration $\theta_{5}$ will be the angular displacement of link $\mathrm{L}_{51}$ with respect to link $\mathrm{L}_{45}$. The coordinate transformation matrix $\mathrm{T}_{5}{ }^{1}$ of joint position 1 with respect to joint position 5 , will be derived by considering both translation and rotation of the coordinate axis. The translation to be considered along $\mathrm{x}$-axis is $-l_{6} \mathrm{C}_{5} \mathrm{~S}(\eta / 2)$, along y-axis $l_{6} \mathrm{C} \theta_{5} \mathrm{~S}(\eta / 2)$, and along $\mathrm{z}-$ axis $l_{6} \mathrm{~S} \theta_{5}$, followed by the rotation of $\theta_{5}$ about $\mathrm{x}$-axis and $\eta$ about $\mathrm{z}$-axis. The co-ordinate transformation matrix $\mathrm{T}_{0}{ }^{1}$ of point 1 with respect to the (origin) fixed frame $\mathrm{F}$,

$\mathrm{T}_{0}{ }^{1}=\mathrm{T}_{0}{ }^{5} \mathrm{~T}_{5}{ }^{1}=\left[\begin{array}{llll}u_{11} & u_{12} & u_{13} & u_{14} \\ u_{21} & u_{22} & u_{23} & u_{24} \\ u_{31} & u_{32} & u_{33} & u_{34} \\ u_{41} & u_{42} & u_{43} & u_{44}\end{array}\right]$

and $\left[\begin{array}{c}X_{1} \\ Y_{1} \\ Z_{1}\end{array}\right]=\left[\begin{array}{c}u_{14} \\ u_{24} \\ u_{34}\end{array}\right]=\left[\begin{array}{l}f\left(\theta_{1} \theta_{2} \theta_{3} \theta_{4} \theta_{5}\right) \\ f\left(\theta_{1} \theta_{2} \theta_{3} \theta_{4} \theta_{5}\right) \\ f\left(\theta_{1} \theta_{2} \theta_{3} \theta_{4} \theta_{5}\right)\end{array}\right]$

where

$$
\begin{aligned}
& u_{14}=-t_{11} l_{6} C \theta_{5} S(\eta / 2)+t_{12} l_{6} C \theta_{5} C(\eta / 2) \\
& +t_{13} l_{6} S \theta_{5}+t_{14} \\
& u_{24}=-t_{21} l_{6} C \theta_{5} S(\eta / 2)+t_{22} l_{6} C \theta_{5} C(\eta / 2) \\
& +t_{23} l_{6} S \theta_{5}+t_{24} \\
& u_{34}=-t_{31} l_{6} C \theta_{5} S(\eta / 2)+t_{32} l_{6} C \theta_{5} C(\eta / 2) \\
& +t_{33} l_{6} S \theta_{5}+t_{34} \\
& u_{41}=t_{41} C \eta+t_{42} S \eta C \theta_{5}+t_{43} S \eta S \theta_{5}
\end{aligned}
$$

The point $f 3$ is situated on the link $L_{51}$. As $L_{51}$ is the reference link there is no rotation of co-ordinate at point $\mathrm{f} 3$ with respect to co-ordinate at point 1 . The coordinate transformation matrix $\mathrm{T}_{1}{ }^{\mathrm{f3}}$ of finger position $\mathrm{f} 3$ with respect to joint position 1 , will be derived by considering only translation and no rotation of the co-ordinate axis. The translation to be considered along $\mathrm{x}$-axis is $-l_{\mathrm{f5}} \mathrm{S}\left(\rho_{5 \mathrm{f}} / 2\right)$, along y-axis $-l_{\mathrm{f} 5} \mathrm{C}\left(\rho_{5 \mathrm{f}} / 2\right)$ and along z-axis 0 .

$$
\mathrm{T}_{0}{ }^{\mathrm{f3}}=\mathrm{T}_{0}{ }^{1} \mathrm{~T}_{1}{ }^{\mathrm{f} 3}=\left[\begin{array}{cccc}
1 & 0 & 0 & -l_{f 5} S\left(\rho_{5 f} / 2\right)+r \\
0 & 1 & 0 & -l_{f 5} C\left(\rho_{5 f} / 2\right) \\
0 & 0 & 1 & 0 \\
0 & 0 & 0 & 1
\end{array}\right] \text { and }
$$

$$
\left[\begin{array}{c}
X_{f 3} \\
Y_{f 3} \\
Z_{f 3}
\end{array}\right]=\left[\begin{array}{c}
-l_{f 5} S\left(\rho_{5 f} / 2\right)+r \\
-l_{f 5} C\left(\rho_{5 f} / 2\right) \\
0
\end{array}\right]
$$

\section{Intersection of the three finger planes}

As the moving link start to rotate about the joint axis, different link configuration arises with different value of relative angular position of the links. The finger planes at the finger position $\mathrm{f} 1$ and $\mathrm{f} 2$ intersect with each other at various position of the links $\mathrm{L}_{12}$ and $\mathrm{L}_{45}$. Figure 6 shows that finger plane o $\mathrm{fl} f \mathrm{ft} 1 \mathrm{p}$ and finger plane o f2 ft2 $\mathrm{p}$ intersects each other and the intersection line is o $\mathrm{i}$. The corresponding angle of the moving link $\mathrm{L}_{12}$ and $\mathrm{L}_{45}$ with the fixed link is $\theta_{1}$ and $\theta_{5}$. There will be different intersection line of the finger planes at different link configuration as $\theta_{1} \& \theta_{5}$ changes. And there will also be the configurations where the plane of the three fingers ( $\mathrm{fl}, \mathrm{f} 2$, f3) will intersect at one line. Figure 7 shows that the three finger planes o f1 ft1 $\mathrm{p}_{1}, \mathrm{o} \mathrm{f} 2 \mathrm{ft} 2 \mathrm{p} 2 \& \mathrm{f} 3 \mathrm{ft} 3 \mathrm{p} 3 \mathrm{p} 03$ intersect in one line oe. It shows the fact that the fingers (f1, f2 \& f3) will be able to achieve different grasp configuration with the fixed thumb (finger 3 ) plane.

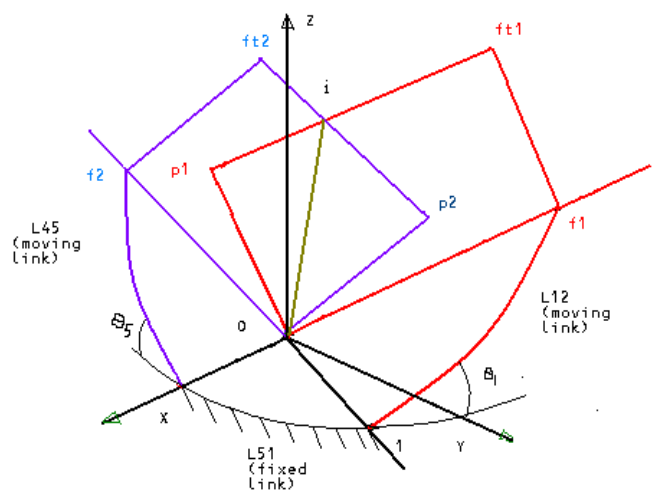

Figure 6: Intersection of the two-finger plane

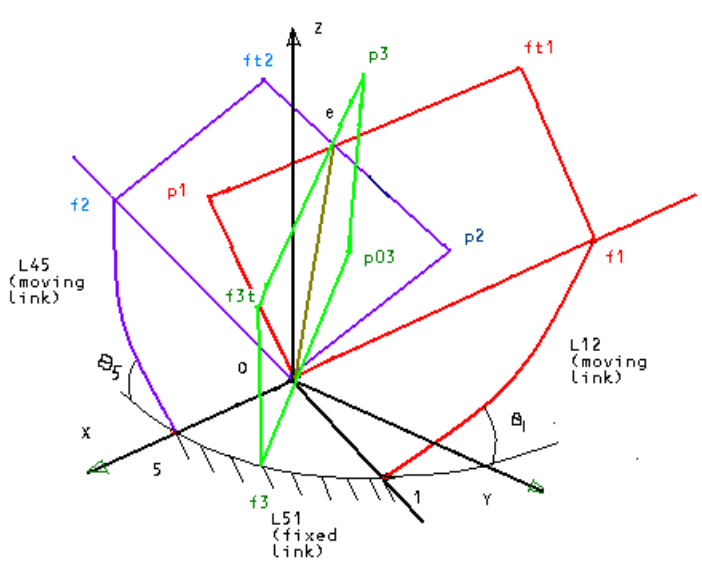

Figure 7: Intersection of the three-finger plane

Journal of Mechanical Engineering, vol. ME36, Dec. 2006

Transaction of the Mech. Eng. Div., The Institution of Engineers, Bangladesh. 
A mathematical formulation of the intersection line oe of the three finger ( $\mathrm{fl}, \mathrm{f} 2 \& \mathrm{f} 3$ ) planes can be derived. One point of the intersection line always lies in the origin 0 . Lets define the other point as $\mathrm{e}$ in the coordinate system as $\mathrm{e}\left(\mathrm{e}_{\mathrm{x}}, \mathrm{e}_{\mathrm{y}}, \mathrm{e}_{\mathrm{z}}\right)$. The direction cosines $(l, m, n)$ of the intersection line oe are

$l=\cos \varphi_{\mathrm{x}}=\mathrm{e}_{\mathrm{x}} / \sqrt{ }\left\{\left(\mathrm{e}_{\mathrm{x}}\right)^{2}+\left(\mathrm{e}_{\mathrm{y}}\right)^{2}+\left(\mathrm{e}_{\mathrm{z}}\right)^{2}\right\}$
$m=\cos \varphi_{\mathrm{y}}=\mathrm{e}_{\mathrm{y}} / \sqrt{ }\left\{\left(\mathrm{e}_{\mathrm{x}}\right)^{2}+\left(\mathrm{e}_{\mathrm{y}}\right)^{2}+\left(\mathrm{e}_{\mathrm{z}}\right)^{2}\right\}$
$n=\cos \varphi_{\mathrm{z}}=\mathrm{e}_{\mathrm{z}} / \sqrt{ }\left\{\left(\mathrm{e}_{\mathrm{x}}\right)^{2}+\left(\mathrm{e}_{\mathrm{y}}\right)^{2}+\left(\mathrm{e}_{\mathrm{z}}\right)^{2}\right\}$

Here the direction angles $\varphi_{x}, \varphi_{y}, \varphi_{z}$ are the angles that the vector oe makes with the positive $x$-, $y$ - and $z$-axes, respectively. In formulas, it is usually the direction cosines that occur, rather than the direction angles, where, $\left(\cos \varphi_{\mathrm{x}}\right)^{2}$ $+\left(\cos \varphi_{\mathrm{x}}\right)^{2}+\left(\cos \varphi_{\mathrm{x}}\right)^{2}=l^{2}+m^{2}+n^{2}=1$.The intersection line pass through the origin o. The equation represents that intersection line is $\quad x / l=y / m=z / n$; where, $l, m, n$ are the direction cosines as described in the above equations.

\section{Link Configurations}

The designed five-bar metamorphic spherical mechanism has the ability to achieve to achieve different link configurations depending on the value of the relative angular displacements $\theta_{1}, \theta_{2}, \theta_{3}, \theta_{4}$ and $\theta_{5}$. By changing these relative angular displacements the robot palm will be able to configure itself in such a way that it can grasp different shape objects by adapting with the environmental conditions. The link configuration states can be described from the mathematical and geometrical model of the fivebar metamorphic spherical mechanism.

According to the spherical link movement there will be relative angular displacement of the links relative to each other as shown in the Figure 8. $\theta_{1}$ represents the angular displacement between the links $\mathrm{L}_{51}$ and $\mathrm{L}_{12}$. Similarly $\theta_{2}$ is the angular displacement between the $\mathrm{L}_{12}$ and $\mathrm{L}_{23}, \theta_{3}$ is between the $\mathrm{L}_{23}$ and $\mathrm{L}_{34}, \theta_{4}$ is between the $\mathrm{L}_{34}$ and $\mathrm{L}_{45}$ and $\theta_{5}$ is between the $\mathrm{L}_{45}$ and $\mathrm{L}_{51}$. The mechanism has been designed in such a way that link $\mathrm{L}_{23}$ and $\mathrm{L}_{34}$ can align with each other. The five-bar spherical mechanism will turn to a four-bar spherical configuration when link $\mathrm{L}_{23}$ will have an angular displacement of $180^{\circ}$ relative to link $\mathrm{L}_{34}$. The links containing the fingers will be able to achieve a good range of configuration state according to design because of its metamorphic ability.

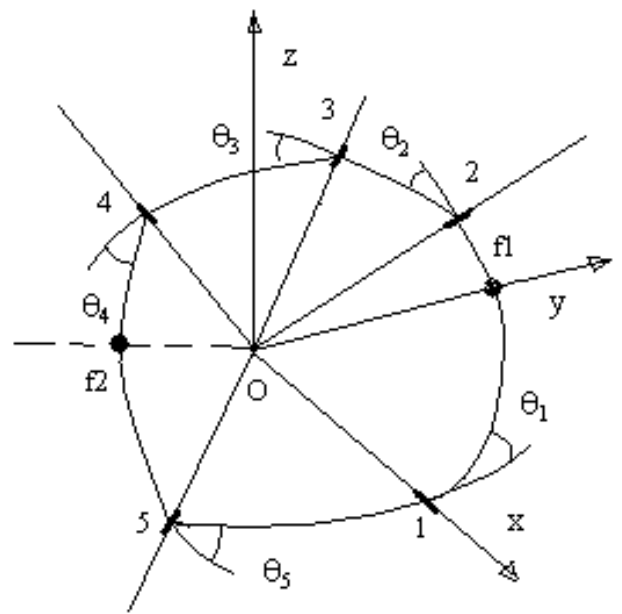

Figure 8: Relative angular displacement of the spherical links
The different combinations of the relative angular displacements $\theta_{1}, \theta_{2}, \theta_{3}, \theta_{4}$ and $\theta_{5}$ of various links can achieve different configurations of the mechanism. Because of the close loop nature of the mechanism there will be certain constraints of the five-bar spherical mechanism. From the mathematical and geometrical analysis some equations have been generated which have to be satisfied by the various configuration stage of the mechanism.

Equation (1) and equation (11) both derived the same coordinate points at position $1\left(\mathrm{X}_{1}, \mathrm{Y}_{1}, \mathrm{Z}_{1}\right)$. That is why

$f\left(\theta_{1}, \theta_{2}, \theta_{3}, \theta_{4}, \theta_{5}\right)=\mathrm{r} \quad ; \quad f\left(\theta_{1}, \theta_{2}, \theta_{3}, \theta_{4}, \theta_{5}\right)=0 \quad ;$ $f\left(\theta_{1}, \theta_{2}, \theta_{3}, \theta_{4}, \theta_{5}\right)=0$

From equation (3) $\mathrm{X}_{2}=f\left(\theta_{l}\right), \mathrm{Y}_{2}=f\left(\theta_{l}\right)$ and $\mathrm{Z}_{2}=f\left(\theta_{l}\right)$ and from equation (23)

$\mathrm{X}_{4}=f\left(\theta_{1}, \theta_{2}, \theta_{3}\right), \mathrm{Y}_{4}=f\left(\theta_{1}, \theta_{2}, \theta_{3}\right)$ and $\mathrm{Z}_{4}=f\left(\theta_{1}, \theta_{2}, \theta_{3}\right)$

As the mechanism is the metamorphic mechanism, there will be a new configuration state of the mechanism when $\theta_{3}$, the relative angular displacement between the link $L_{23}$ and $\mathrm{L}_{34}$ will be $180^{\circ}$. At this the five bar mechanism will transform to four bar mechanism and the co-ordinates of point 2 and point 4 will coincide.

Therefore, it can be said that, At $\theta_{3}=180^{\circ}$, equation (8) = equation (23)

$f\left(\theta_{l}\right)=f\left(\theta_{l}, \theta_{2}, \theta_{3}\right) \quad ; \quad f\left(\theta_{l}\right)=f\left(\theta_{l}, \theta_{2}, \theta_{3}\right) \quad \&$ $f\left(\theta_{1}\right)=f\left(\theta_{1}, \theta_{2}, \theta_{3}\right)$, Where $\theta_{3}=180^{\circ}$

The mathematical formulation for different link configurations has been expressed in above equations.

\section{CONCLUSIONS}

A geometrical model and detail kinematic analysis of the five-bar spherical metamorphic mechanism has been presented. The mathematical formulation has been developed to achieve different link configurations and define the position and orientation of any point of the link in three-dimensional space. This model can be used to design a metamorphic robot palm, which can achieve human-like operational flexibility.

\section{REFERENCES}

1. Romdhane, L., Duffy, J.,(1986). Kinestic Analysis of Multifingered Hands. Int. J. Rob. Res. Vol 2. pp . 317.

2. Salisbury, J.K.,(1982). Kinematics and Force Analysis of Articulated Hand. PhD Thesis, Stamford University.

3. Kobayashi, H., (1985). Control and Geometrical Considerations for an Articulated Hand. Int. J. Robot. Res. 4(1).

4. Kerr, J.R., \& Sanger D, J., (1986). Restraint Analysis of a Rigid Body using frictional Elastic Contacts.ASME paper no .86 - DET - 136 . 
5. Kerr, J.R., Analysis of Multifingered Hands, PhD Thesis,Stamford University , Department of Mechanical Engineering.

6. Kerr, J.R., \& Roth, B.,(1986). Analysis of Multifingered Hands. Int. J. Rob. Res. Vol 4, pp. 317.

7. Salisbury,J.K., \& Craig, J.J.,(1981). Articulated Hands: Force Control and Kinematic Issues, Robotic Research, Vol 1, No 1, pp. 1-15.

8. Dai, J.S., \& Rees Jones, J.,(1999) .Configuration Transformations in Metamorphic Mechanisms of Foldable/ Erectable Kinds . Proc. of $10^{\text {th }}$ World Congress on the Theory of Mechanisms, Oulu , Finland, Vol 2 , pp. $542-547$.

9. Dai , J.S., \& Shah, P., (2003). Orientation capability of Planner Manipulators Using Virtual Joint Angle Analysis. Mechanism and Machine Theory, Vol 38(3), pp. $241-252$.
10. Dai, J.S., \& Rees Jones, J.,(1999). Mobility in Metamorphic Mechanisms of Foldable/ Erectable Kinds. Journal of Mechanical Design Transaction of ASME, Vol 121 (3), pp. 375 - 382.

11. Dubey, V. N., Dai, J. S., Stamp, K.J., \& Rees Jones, J., (1999). Kinematic Simulation of a metamorphic Mechanism. $10^{\text {th }}$ World Congress on Theory of Machine and Mechanism, pp. $98-103$.

12. McCarthy, J. M., (2000) Geomatric Design of linkage, $1^{\text {st }}$ edition, Springer.

13. Duffy, J., (1980) Analysis of Mechanisms and Robot Manipulators, Wiley. 